\title{
Mobile Design Guidelines in the Context of Retail Sales Support
}

\author{
Rainer Blum, Karim Khakzar \& Werner Winzerling \\ Fulda University of Applied Sciences \\ Germany
}

\section{Introduction}

Mobile technology is an inherent part of today's private and professional life in the developed nations, with mobile telephony at the forefront. Optimization of business processes with mobile technology presents a topic of interest for enterprises for several years focusing primarily on the sales force and logistics (Wichmann \& Stiehler, 2004).

Though still rare in practice, by supporting the duties of the retail sales staff in their daily personal sales activities, mobile technology is assumed to generate added value in the retail context for customers, sales staff and shop owners as well. Individual innovative companies still test these techniques, for example the METRO Group with its "Future Store Initiative". But, also in a broader context and independently of today's enormous diffusion of mobile technology, mobile design still remains an area of active research.

In this regard, employing mobile devices for the retail sales support at the point of sale was a subject in the research project IntExMa, "Interactive Expert System for Made-to-measure Clothing" at Fulda University of Applied Sciences. In doing so, usability was regarded as a fundamental quality criteria.

Many standards, guidelines and scientific papers document the existing usability knowledge in the field of HCI. While this expertise has predominantly evolved in the desktop computer domain over a period of several decades, interface design for mobile services is still regarded to be "in its infancy" (USE-ME.GOV, 2004). Yet, only a few scientific and hardly any generally accepted guides for mobile interface design exist. These are naturally not based on such a broad basis of experiences than in the desktop domain.

Gong \& Tarasewich propose a range of usability guidelines for the mobile devices domain (Gong \& Tarasewich, 2004). They start from Shneidermans \& Plaisants eight "Golden Rules", implicitly termed desktop-oriented, and base them on their own and other research findings. Many other studies concentrate exclusively on the optimized display of web pages on handheld devices, e.g. (Kärkkäinen \& Laarni, 2002). The two major companies offering operating systems for handhelds, Palm Source and Microsoft, have each published their own more or less product specific guidelines (see below).

\subsection{Objectives and Significance}

Facing the heap of well-established desktop-descending guidelines, standards and recommendations, like e.g. the outstanding ISO 9241, one objective of this work is to answer 
the question if these resources are also relevant and applicable for the mobile context. In addition, existing mobile guidelines are also taken into account.

Putting it all together, a compilation of design principles is proposed, each rationally explained and detailed with a range of exemplary, more concrete rules and clarifying examples. Thus, we aim to contribute to the state-of-the-art by interpreting existing, mostly desktop-oriented guidelines under a new point of view. In doing so, the lack in the mobile interface design domain shall be attenuated.

All recommendations are specifically shaped for the context of use at hand: support of personnel selling activities at the point of sale with handheld technology in the hands of the sales persons.

\subsection{The IntExMa Sales Support System}

The basic concept of the IntExMa system is to effectively support in-store, "real world" sales processes with e-commerce technology ("virtual shopping"), thus generating added value for customers, sales staff and shop owners. Made-to-measure shirts constitute the particular sales context to which the system is adapted. According to the customers' body measures, a 3D figure is automatically generated and dressed in the desired shirt applying a highly realistic physical simulation. Customers are able to use a subset of the system's interactive features via a simple custom-made knob and buttons interface.

Via a PDA, the salesperson has mobile access to the entire functionality of the system: electronic product catalogue with capacious ordering functions, customer data, and 3D scene interaction. This includes interaction with the stationary display showing the "virtual try-on" via the PDA. Within this scope we investigated to what extent mobile devices can support the duties and responsibilities of the retail sales staff in their daily personal selling activities.

\section{Method}

Several distinct steps were taken in order to gather the necessary theoretical basis to be able to compile the desired specific collection of design guidelines. They were chosen following ISO 13407 (ISO 13407, 1999).

First, the specific characteristics of handheld computing and the PDA device class were analyzed and compared with the desktop computing domain. Thereby, the manifold possibilities concerning interaction techniques and forms became apparent but also the limitations to be considered were diagnosed. Then, a detailed context of use analysis was conducted and accordant requirements were formulated.

Apart from that, some of the most important and accepted usability standards, guidelines and scientific papers were analyzed in order to assess which insights are also valid for the mobile domain and can be transferred into the present context.

Taking these results as a theoretical basis, finally, the desired compilation of design principles, concrete rules and clarifying examples was developed.

This project specific standard was applied for the development of mobile applications for the aforementioned IntExMa system. Usability evaluation of these implementations will imply also the indispensable evaluation of the developed guidelines. 


\section{Context of Use and Requirements}

The context of use analyses was conducted in-depth following ISO 13407 (ISO 13407, 1999). Some major points are reproduced in the following.

Sales support as a central activity is characterized by a broad spectrum of tasks aiming to enhance sales. This study focuses on the domains of the retail business not dominated by self-service but where consultation-intensive products are sold and the customers are served by well-trained personnel. This personal selling is primarily characterized by a direct communication between customer and sales person.

\subsection{User Characteristics, Tasks, Environment}

Sales process support in the retail business is characterized by a high heterogeneity concerning users' respectively salespersons' characteristics - gender, age, education, experiences, knowledge, etc.

Skills and knowledge about the business processes and methods to be supported can be estimated as comprehensive. Furthermore, experiences with PDA usage are expected to be very low, but to substantially rise with the usage period. Previous knowledge concerning interaction techniques and modes may exist but can't be assumed for all users. Using a portable touch screen of this size is certainly novel for most of them. A short, e.g. one-day training on the application is expected.

Tasks groups to be supported by the mobile application are informing and giving advice, utilizing and administering customer data, processing payments, providing service and sales preparation and follow-up. The present context of use is characterized by a "hierarchy of tasks" like described by Gorlenko \& Merrick (Gorlenko \& Merrick, 2003): During interaction, the tasks external to the device are in the centre of interest while system-internal tasks, e.g. entering a search keyword, accrue additionally. Also, the social environment only provides minimum freedom for interaction with the mobile device, as mostly the customer is in the focus of the attention of the personnel.

\subsection{Resulting Requirements}

Following the results of the context of use analysis, an important requirement is to design the system for users with diverse experiences, skills and knowledge concerning information technology and to cater for novices as well as for "power users". In order to reduce the acceptance threshold users must be enabled to acquire basic functionality by themselves.

Seamless integration into the existing environment supports the flexible functionality changeover of sales persons during work.

The customer must be taken into account as "indirect user" constantly present during service and with needs taking the centre stage of personnel selling. Therefore, the demand of resources, e.g. of attention or time, of the application should be as small as possible. Onehand or, even better, hands-free operation of the device is desirable in order to keep interacting with real objects as much as possible.

The users should - only to a small extent - have to "make place“ for the device operation. Their imposed mental workload should be kept low, while simultaneously staying informed about the current process status, despite frequent interruptions.

Also, the sales person should have the possibility to present output information visually to the customer, e.g. if the "spoken word" is not sufficient due to product complexity or lack of the completed product (e.g. mass customization). 
The mobile interactive system must provide required information on the spot and in the desired level of detail with flexible search and meaningful comparison options. In addition, a methodical and controlled proceeding of the sales person must be supported and ensured. Where possible, interaction with physical objects, e.g. via product bar codes, as "information bearers" should be possible, in order to avoid unnecessary searching in electronic resources and to further enhance integration with the real environment.

\section{Existing Guidelines}

A selection of the most important representatives of desktop-related standards and guidelines was analyzed concerning their relevance and their transferability for the handheld/PDA domain.

The most well-known user interface design standard is probably ISO 9241 (ISO 9241, 19932002). Parts 2, 10 and 11 can be fully applied. In part 12 only the addressed window technique is subject to severe limitations on the handheld platform. User guidance in part 13 is absolutely relevant, with reservations concerning the extent and design of online help due to limited display space. Part 14 has relevance, though only small-sized menu dialogues make sense on PDAs. Command dialogues in part 15 are hardly relevant, unless it comes to speech control. Many recommendations for the direct manipulation concept in part 16 are applicable though the graphical representation and the manipulation features of objects and their states should be much less comprehensive than on the desktop. Furthermore, direct manipulation beyond basic interaction (e.g. dragging objects) demands to much concentration from the user to be reasonably applied in the mobile context (Kristoffersen \& Ljungberg, 1999). Part 17 is again mostly of high relevance for the handheld domain. Some of the recommendations imply a cursor. Though existent for form fields and text documents, mouse pointers are not standard. They may be activated in certain operating systems or simulated by applications.

ISO 14915 (ISO 14915, 2002-2003) is basically relevant for handheld design, due to similarity to ISO 9241 though it must be considered that the multimedia capabilities of these devices do not approximate those of multimedia desktop computers.

The analyzed "Ten Usability Heuristics" by Nielsen (Nielson, 1994) and eight "Golden Rules" by Shneiderman \& Plaisant (Shneiderman \& Plaisant, 2004) are valid for handheld design, as they are independent of specific technologies and device form factors. They must be individually interpreted in order to apply them practically in a specific context of use.

The basic principles for user interface design and many of the more concrete guidelines and rules presented in the "desktop-descending" IBM Common User Access (CUA) Guidelines, refer to (IBM Corporation) for a condensed presentation, in the Microsoft Official Guidelines for User Interface Developers and Designers (Microsoft Corp, 1999) and in the Apple Human Interface Guidelines (Apple Computer, Inc., 2005) are applicable for the present setting.

The Windows Mobile Guidelines (Microsoft Corp., 2005) and the Palm OS User Interface Guidelines (PalmSource, Inc., 2003) are the only available more comprehensive mobile guidelines. Here, Microsoft does not provide basic design principles but some more general guidelines which are converted into very practical Windows Mobile specific recommendations. The Palm starts with basic principles, moves on to concrete design guidelines and ends with detailed technical layout guidelines. Main focus from the point of 
view of functionality is the Personal Information Manager (PIM) domain, e.g. administrating contact details, dates, tasks, notes or even documents. So, practical applicability is ensured in both cases. Both compilations contain relevant information also for the context of use at hand.

\section{Specific Design Guidelines Compilation}

Based on the insights and recommendations in the previous chapters, seventeen design guidelines are presented in the following.

Every principle consists of a short title, followed by a more detailed wording as a complete sentence in the first paragraph. As interpreting and applying these principles first requires to fully understand their logical foundation, the second paragraph contains accordant explanations. Then, for lack of space, one exemplary of several rules is listed, that focus specific design questions. The reproduction of concrete design examples and the listing of references to existing standards and guidelines is abandoned here. In the full research report (Blum, 2006) this serves the underpinning of the scientific significance of each principle and its corresponding rules as well as pointer to further resources.

\subsection{Choose "Low-absorbing" Interaction Techniques}

Design the system in such way, that its use occupies the user's manual resources and sensory functions (vision, hearing, perception in general) as little as possible.

Interaction with an application draws upon the sense of the user and requires manual activity: The system's output has to be perceived visually, acoustically, potentially also tactilely, processed in the brain and subsequently converted into motor reactions. Resources tied up in this process are at the same time required for the primary tasks of the sales person in the sales process support. Due to the importance of non-verbal communication for the personnel selling, the visual senses are highly significant.

An exemplary rule is to make the operation of the system possible without the usually obligatory pen and, even better, with only one hand.

\subsection{Design on the Basis of the Real Tasks}

When designing the user interface, refer primarily to the requirements of the tasks to be completed in the real environment and the requirements of the context of use.

Thereby, the components of the user interface shall "take a back seat" in the perception of the user. An additional charge of the sales person with system-internal tasks during the customer dialogue should be preferably avoided. Also, frustration by reason of inability to tackle tasks adequately is counteracted.

To choose terminology, symbols and metaphors according to the real tasks and, as such, to build on existing knowledge of the user, is one concrete rule.

\subsection{Design for Short, Frequently Interrupted Tasks}

Map tasks in such a way that the separate steps may be processed in a flexible order and action sequences may be interrupted and resumed later.

The hardly to plan course of a customer dialogue demands for a variable but thorough completion of the separate work tasks (task sequence) and a spontaneous and flexible response to the remarks and requests of the customer. The interactive system needs to show 
the same degree of flexibility while ensuring a well-structured advancement and an exhaustive task processing.

One exemplary corresponding rule is to provide the processing status of a task "at a glance", concretely by presenting all necessary information in one screen form and indicating data fields that are already treated.

\subsection{Strive for Consistency}

Question all user interface components whether they can be designed consistent to the experience of the sales personnel, other employed applications and among each other.

Consistency enables the users to apply existing knowledge and to quickly build a mental model of the application's functional concept. They come across familiar objects and training period can be reduced. Thereby, the user interface should furthermore be operated intuitively and without surplus mental load and additional concentration effort. Consistent positioning of controls enables the salesperson to operate the application "blindly" (without concentrating on the display).

An exemplary rule is to rarely make exceptions but in situations that justify their use - and to design them coherently.

\subsection{Cater for Easy and Intuitive Interaction}

Give interactive elements a design as simple as possible and make sure, that the user intuitively detects how to interact with them.

Controls, whose operation is apparent should result in a faster learnability and a lower mental workload for the user. If, in addition, the interaction techniques are straightforward, the application should demand less attention from the user.

Exemplary rule: For text fields, make clear which kind of input is expected.

\subsection{Offer Instantaneous, Informative Feedback}

Provide a feedback for every interaction, that, with its "magnitude", reflects appropriately the significance and frequency of occurrence of the particular action and that considers the context of use.

Prompt reaction to the user's interaction supports a sense of controllability. On the one hand, users are informed, that their input was recognized, on the other hand the "new" system state is communicated. Panic, that may occur due to too long response times when pressure to perform is high, can be avoided. A suitable degree of clarity and demand of attention must be ascertained.

A rule is to acknowledge every interaction with a prompt feedback.

\subsection{Provide Simple and Unambiguous Navigation}

Design navigation instruments in such a way, that the user can answer the questions "Where am I, how did I get here?", "What can I do here?", "Where can I go next?" at all times.

Due to frequent interruptions, the salespersons must be enabled to recognize at a glance at which position in the task sequence they are currently. Also, it must be transparent how 
they got there (or what the previous steps were) and which options to proceed are offered, i.e. which elements are available for follow-up interaction.

For example, an option for navigating backward and, if applicable, forward in a list of lastly visited dialogue pages should be provided.

\subsection{Cater for Effective, Usable Content}

Prepare content (product data, customer data etc.) and structure the design in such a way, that an effective handling by the salespersons is ensured and that, at the same time, their limited attention is considered.

Parts of the information taken from the PDA is passed on to the customer, partly it is needed for processing the more formal steps of the sales process. Preparation and reproduction of information must be oriented along this process and has to consider that the salespersons have to pay the bigger part of their attention to the customer.

One corresponding rule is to make it possible to output content otherwise than via the PDA display (e.g. graphically as product comparison chart), in order to communicate it to the customer not only verbally.

\subsection{Design Well-defined Dialogues}

Structure tasks in small action groups with obvious start and end; inform the user explicitly about their successful completion.

In a working environment characterized by lots of disruption and distraction, the explicit confirmation about a successfully completed (even small) working process creates a feeling of release and satisfaction by the user. The user can tick off one item on his mental task list and focus on the remaining steps.

An exemplary rule is to structure tasks such that they can be "displayed" on a single screen page.

\subsection{Reduce Functionality to its Minimum}

Reduce the variety of the application's functions to the minimum mainly required by the salesperson during the customer dialogue conducted mobile.

The fewer functions need to be accommodated in the application, the higher are the chances to achieve a good usability. This can lead to a clearer structure and the possibility to dimension control elements more generously. These facts should in turn reduce the practical training for the salesperson, as well as the cognitive load and the demand for concentration. Too many details can obfuscate the structure, complicate the conceptual model on the user's part and produce confusion - especially if the users cannot pay most of their attention to the application.

One corresponding rule is to not try to port a desktop application - in the sense of transforming it with only minimal adjustments.

\subsection{Consider Different User Characteristics}

Try to cover a wide spectrum of user characteristics by providing alternative possibilities of interaction. 
Even in a small shop you will find different kinds of characteristics amongst the salespersons, e.g. regarding perception-related abilities, motor coordination skills and cognitive capacity or education, knowledge, abilities and experiences. These differences can be considered with a thoroughly designed device.

Exemplary rule: Alternative interaction paths and short cuts should be offered.

\subsection{Ensure Easy and Permanent Access to Important Options}

The availability of important and frequently used options should vary only little and access to these options should be eased explicitly.

Learnability decreases and cognitive load increases if certain command options are available in some, but not in all situations. Such variations can cause discontent and concern, and potentially complicate the user's mental model of the application.

An exemplary rule is to avoid operation modes, particularly as their marking requires additional status messages.

\subsection{Minimize the Quantity of Required Interaction and Input}

Demand as little interaction from the user as possible and take advantage of already available information.

A low quantity of required elementary interactions, which in addition require only little concentration, should keep the salesperson's stress low. The intensive usage of data that is already available in the system and respectively in the shop, reduces the necessity to reproduce information from memory.

For example, meaningful standard values should always be provided, that constitute a basis for further processing.

\subsection{Prevent Errors}

Analyze which errors can occur. Indicate which error situations can arise or better, eliminate those from the very beginning.

The present context of use with frequent disruptions of the work process is susceptible to operator errors. The salespersons should have the impression that the handling of the PDA is "foolproof" and that they can hardly make a mistake if at all. This feeling should reduce their mental stress.

A corresponding rule is to explicitly indicate unsaved data in order to avoid accidental discarding.

\subsection{Offer Straightforward Error Recovery}

Indicate errors to users and support them during the recovery process.

The possibility to easily recover from errors is a precondition for a self-confident operation of an application by the user. This leads to less mental workload for the salesperson as well as a lower demand of attention during a potentially error-prone stress situation. Being able to cancel a previous action in case of an unexpected reaction of the application can avoid frustration.

Rule: Cancelling of actions should be offered with different granularities, from elementary interaction steps (e.g. not releasing a pressed button) to complete task sequences. 


\subsection{Offer Concise, Context-sensitive Help}

Offer an online help corresponding clearly and briefly to the current dialogue context.

The leading principle should be that the system is usable without documentation. Nevertheless, the users should be able to recall help, which refers to the current dialogue situation. This enables them to self-help even during the customer dialogue. Comprehensive help should be offered via the manual or separate learning software.

To specify concrete actions in the help information is a corresponding rule.

\subsection{Design Easily Perceivable, Readable, Rich in contrast and Aesthetic Dialogues}

During the design process, aspire after clearness, simplicity, visual attractiveness and a harmonic overall picture.

On the one hand, the goal is to facilitate the perception and handling of the available information by the salesperson. Variable environmental characteristics (e.g. changing lighting conditions) should not have a negative influence. On the other hand, the visual design should appeal to the users and animate them to use the device.

Example rule: Icons should be simple and rich in contrast and have clear contours.

\section{Conclusions}

The aim of this chapter was to contribute to the development of guidelines for mobile (handheld respectively PDA) design, a domain not yet sufficiently covered especially when it comes to business process support. A rather complete set of principles and exemplary rules is presented, based on analysis, interpretation and amendment of established guidelines for desktop user interfaces and some of the rare mobile design guides.

The question if desktop-descending guidelines, standards and recommendations are also relevant and applicable for the mobile context could be answered positively for a range of well-established representatives. It is important to notice, that the presented guidelines compilation is adapted to the specific context of use at hand: handheld support for the sales person at the point of sale being involved in personnel selling. Though this setting restricts some of the variable factors to be considered, its heterogeneity is still so high, that applying these guidelines requires a thorough consideration of the specific situation. Therefore, this work should be generally useful to practitioners and HCI researchers.

It proved to be challenging to find a balance between complete coverage of all relevant aspects and treatment of each in sufficient level of detail. Starting from basic principles, explaining their rationale, providing several exemplary, more concrete rules and giving concrete examples is the proposed way to ensure practical applicability but to also allow to assess and integrate new developments.

Evaluation by application and analysis in concrete implementations and the consideration of further related quality aspects, e.g. accessibility or joy of use, are options for next steps.

\section{Acknowledgments}

This research was financially supported by the German Federal Ministry of Education and Research within the framework of the program "Angewandte Forschung an Fachhochschulen im Verbund mit der Wirtschaft" $\left(\mathrm{FH}^{3}\right)$. 


\section{References}

Apple Computer, Inc. (2005). Apple Human Interface Guidelines, http:/ / developer.apple.com/documentation/UserExperience/Conceptual/Apple HIGuidelines/OSXHIGuidelines.pdf, Last retrieved 16/05/08

Blum, R. (2006). Gestaltungsempfehlungen für PDA-Benutzungsschnittstellen am Beispiel der Verkaufsunterstützung im Einzelhandel, Master Thesis, Fulda University of Applied Sciences

Gong, J. \& Tarasewich, P. (2004): Guidelines for Handheld Mobile Device Interface Design, Proceedings of the 2004 Decision Sciences Institute (DSI) Annual Meeting, pp. 37513756, ISBN 0966711807, Boston, Nov. 2004, Decision Sciences Institute, Atlanta

Gorlenko, L. \& Merrick, R. (2003). No wires attached: Usability challenges in the connected mobile world, In: IBM Systems Journal, Vol. 42, No. 4, pp. 639-651

IBM Corporation: Design Principles, Last retrieved 16/05/08 https://www.306.ibm.com/software/ucd/designconcepts/designbasics.html

ISO 13407 (1999). Human-centred design processes for interactive systems

ISO 14915 (2002-2003). Software ergonomics for multimedia user interfaces, Parts 1-3.

ISO 9241 (1993-2002). Ergonomic requirements for office work with visual display terminals (VDTs), Parts 2, 10 to 17

Kärkkäinen, L. \& Laarni, J. (2002): Designing for Small Display Screens, Proceedings of the second Nordic conference on Human-computer interaction, pp. 227-230, Aarhus, Oct. 2002, ACM Press

Kristoffersen, S. \& Ljungberg, F. (1999): „Making Place“ to Make IT Work: Empirical Explorations of HCI for Mobile CSCW, Proceedings of the International ACM SIGGROUP Conference on Supporting Group Work, pp. 276-285, ISBN 0897910651, Phoenix, Nov. 1999, ACM Press

Microsoft Corp. (1999). Official Guidelines for User Interface Developers and Designers, Microsoft Press, ISBN 0735605664, Redmond

Microsoft Corp. (2005). Design Guidelines, Windows Mobile Version 5.0 SDK, Last retrieved 16/05/08 http://msdn.microsoft.com/en-us/library/ms879586.aspx

Nielson, J. (1994). Heuristic evaluation, In: Usability Inspection Methods, Nielson, J. \& Mack, R.L. (Eds.), pp. 25-62, John Wiley \& Sons, ISBN 0471018775, New York

PalmSource, Inc. (2003). Palm OS User Interface Guidelines, http://www.accessdevnet.com $\angle$ docs/ uiguidelines.pdf, Last retrieved 16/05/08

Shneiderman, B. \& Plaisant, C. (2004). Designing the User Interface - Strategies for Effective Human-Computer Interaction, 4th ed., Addison Wesley, ISBN 0201694970, Amsterdam

USE-ME.GOV (2004). Review of State of the Art in User Interface Design for Mobile Applications, Deliverable D4.1

Wichmann, T. \& Stiehler, A. (2004). Prozesse optimieren mit Mobile Solutions, Berlecon Research $\mathrm{GmbH}$, Berlin 


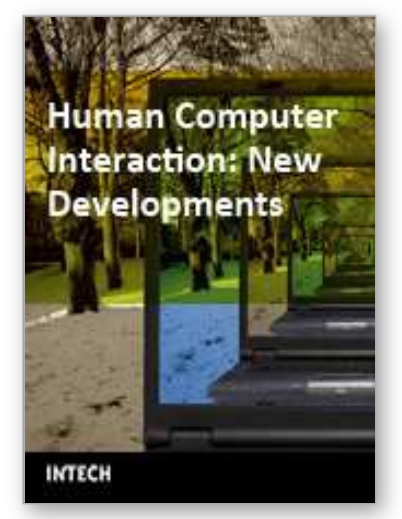

\author{
Human Computer Interaction: New Developments \\ Edited by Kikuo Asai
}

ISBN 978-953-7619-14-5

Hard cover, 382 pages

Publisher InTech

Published online 01, October, 2008

Published in print edition October, 2008

The book consists of 20 chapters, each addressing a certain aspect of human-computer interaction. Each chapter gives the reader background information on a subject and proposes an original solution. This should serve as a valuable tool for professionals in this interdisciplinary field. Hopefully, readers will contribute their own discoveries and improvements, innovative ideas and concepts, as well as novel applications and business models related to the field of human-computer interaction. It is our wish that the reader consider not only what our authors have written and the experimentation they have described, but also the examples they have set.

\title{
How to reference
}

In order to correctly reference this scholarly work, feel free to copy and paste the following:

Rainer Blum, Karim Khakzar and Werner Winzerling (2008). Mobile Design Guidelines in the Context of Retail Sales Support, Human Computer Interaction: New Developments, Kikuo Asai (Ed.), ISBN: 978-953-7619-14-5, InTech, Available from:

http://www.intechopen.com/books/human_computer_interaction_new_developments/mobile_design_guideline s_in_the_context_of_retail_sales_support

\section{INTECH}

open science | open minds

\section{InTech Europe}

University Campus STeP Ri

Slavka Krautzeka 83/A

51000 Rijeka, Croatia

Phone: +385 (51) 770447

Fax: +385 (51) 686166

www.intechopen.com

\section{InTech China}

Unit 405, Office Block, Hotel Equatorial Shanghai

No.65, Yan An Road (West), Shanghai, 200040, China

中国上海市延安西路65号上海国际贵都大饭店办公楼405单元

Phone: +86-21-62489820

Fax: $+86-21-62489821$ 
(C) 2008 The Author(s). Licensee IntechOpen. This chapter is distributed under the terms of the Creative Commons Attribution-NonCommercialShareAlike-3.0 License, which permits use, distribution and reproduction for non-commercial purposes, provided the original is properly cited and derivative works building on this content are distributed under the same license. 